\title{
The Complexities of Depression
}

\author{
Stephen M. Strakowski, MD
}

\section{Keywords}

Major depression; Mood disorders; Alcoholism; Suicide; Antidepressants; Psychiatry

\begin{abstract}
Major depression is one of the world's most common medical problems, being the leading cause of disability in developed nations worldwide and the third overall cause of global disease burden (1). In the United States, approximately $7 \%$ of the population experiences depression each year (2). Although there is some variability in rates of depression among different groups, nonetheless it strikes across ethnic, racial, national and socioeconomic boundaries (3). As a major public health problem, treatment of depression is of paramount importance, yet relatively few advances in treatments have occurred for nearly two decades and available interventions fail to provide timely relief for many affected people (4). New interventions are clearly needed, but to make these advances, a better understanding of the brain basis of depression is necessary. In this section of Current Psychiatry Reports, several leading experts examine aspects of the treatment of depression ranging from specific subgroups (e.g., depression complicated by psychosis) to specific aspects of the illness (namely suicide). To help frame these discussions, the possible underlying functional neuroanatomy of depression is also reviewed.
\end{abstract}

Depression complicates (and is complicated by) a number of different conditions, but one of the more common associations is with alcoholism. As described by DeVido and Weiss (5), alcohol use disorders are nearly three times more common in depressed individuals than the general population and the converse is also true, i.e., that depression is over-represented in people with alcoholism. These patients are more likely to exhibit poor treatment outcomes and all aspects of treatment, from initial assessment to treatment decisions to treatment adherence, are more difficult than in subjects with each condition separately. Historically, treatment of the depressed alcoholic has often focused on one or the other condition first, only to treat the other after the first had resolved. DeVido and Weiss present evidence that integrated treatment, in which both conditions are managed under a single treatment program or team, is the most effective approach. Additionally, integration of both pharmacotherapy and psychotherapy may provide additional benefit (5). Integrated treatment is, unfortunately, not widely available; the arguments presented by DeVido and Weiss suggest that expanding these opportunities could provide a major advance in this common co-occurrence.

Although major depression causes significant morbidity, clearly the most tragic outcome of depression is death by suicide. Suicide prevention is a primary goal in the treatment of anyone with depression, yet even with advances in treatment approaches, it has not been clear whether progress toward this goal is being made. Schwartz-Lifshitz et al (6) observe that although a number of risk factors for suicide, including the presence of major

Address correspondence to Dr. Stephen Strakowski, Dean's Office, University of Cincinnati College of Medicine, 219 Albert Sabin Way (ML0555), Cincinnati, OH 45267-0555. Stephen.Strakowski@uc.edu, Phone: 513-558-4274; fax: 513-558-0187.

Disclosure No potential conflicts of interest relevant to this article were reported. 
depression, have been identified, because these characteristics are generally common and suicide is generally rare, preventing suicide remains challenging. They suggest that prevention of suicide requires not only alert, thorough clinical care, but also community and social participation that includes public education and responsible media coverage in order to impact the prevalence of suicide. Their article reminds us that everyone, not just patient advocacy groups and mental health care providers, are responsible for improving outcomes of people suffering from depression and other mental illnesses.

Depression is variable in its presentation as a number of different symptom complexes occur. Psychotic depression is one such variant, and the occurrence of psychosis during a depressive episode is associated with a worse course of illness. It has been recognized for many years that depression complicated by psychosis is poorly responsive to antidepressant monotherapy, requiring concurrent administration of an antipsychotic (7). However, as discussed by Nelson (7), abnormalities of the HPA-axis observed in psychotic depression suggest alternative approaches that might be effective. In particular, he describes how treatment with the glucocorticoid receptor antagonist mifepristone may be an effective intervention in this subtype of major depression. This novel approach suggests alternative mechanisms toward treatment development that may lead in the future to better interventions in this patient population.

Indeed, I am frequently struck by how, even though I graduated from residency almost 25 years ago, our treatments for major depression have minimally changed since then, remaining primarily linked to modulating monoamine levels, particularly serotonin. Although there is nothing intrinsically wrong with this approach, unfortunately our current treatments fail to provide significant improvement, particularly recovery, nearly half the time (8). As noted, given the public health significance of major depression, novel treatments are desperately needed. Murrough and Charney (8) address this limitation in our antidepressant options, but note promising new leads in drugs that modulate the glutamatergic system. In particularly, the N-methyl-d-aspartate receptor antagonist ketamine provides a model for developing new agents within this system. Perhaps more importantly, ketamine reminds us to reconsider historical notions of the neurobiology of depression to foster development of alternative strategies for treatments beyond the monoaminergic modulation models that have dominated antidepressant development for more than fifty years.

In fact, it is likely that our ability to treat major depression will not dramatically improve until we better understand its neurobiology. Complicating these efforts is the observation that depression appears to arise nonspecifically in response to a wide variety of brain insults ranging from stroke and schizophrenia to infection and traumatic brain injury. This observation suggests that the neurobiology of depression is variable and may be related specifically to its underlying causes that are often difficult to identify. Nonetheless, despite different etiologies, a common end-state does occur (namely depression) which may have a shared neurobiology that could provide a target for treatment development despite the heterogeneity of this condition. Specifically, as outlined by Pandya et al (9), depression does not neatly localize into singular brain regions, but affects distributed corticolimbic networks. However, measurements of the function of components within these networks might offer opportunities to develop objective markers of early treatment response, if not specific locations to apply neuromodulatory treatments such as rTMS (9). Moving from functional neuroanatomic to molecular understanding of the brain pathology underlying depression may provide even better alternatives to guide treatments in the future.

Although major depression remains a far too common, complicated condition to manage, these articles suggest that progress is steadily being made that may ultimately provide 
marked improvements in treatments for this condition. The approaches highlighted - i.e., to focus on specific subgroups, specific behaviors, and novel treatment interventions, while better understanding the neurobiology of the illness - offer promise for these very improvements. With these approaches, we can be optimistic that our options for those patients presenting in our offices with major depression will truly expand so that, in the future, we can over interventions that provide quick, predictable relief. We are hopeful that the articles included in this issue will inspire investigators toward that very progress in order to improve the lives of our patients.

\section{References}

1. World Health Organization. The global burden of disease: 2004 update. 2008

2. Kessler RC, Chiu WT, Demler O, Walters EE. Prevalence, severity, and comorbidity of twelvemonth DSM-IV disorders in the National Comorbidity Survey Replication (NCS-R). Archives of General Psychiatry. 2005; 62:617-27. [PubMed: 15939839]

3. Kessler RC, Berglund PA, Demler O, Jin R, Walters EE. Lifetime prevalence and age-of-onset distributions of DSM-IV disorders in the National Comorbidity Survey Replication (NCS-R). Archives of General Psychiatry. 2005; 62:593-602. [PubMed: 15939837]

4. Rush AJ, Trivedi MH, Wisniewski SR, Nierenberg AA, Stewart JW, Warden D, Niederehe G, Thase ME, Lavori PW, Lebowitz BD, McGrath PJ, Rosenbaum JF, Sackeim HA, Kupfer DJ, Luther J, Fava M. Acute and longer-term outcomes in depressed outpatients requiring one or several treatment steps: A STAR*D report. Am J Psychiatry. 2006; 163:1905-1917. [PubMed: 17074942]

5. Devido JJ, Weiss RD. Treatment of the depressed alcoholic patients. Curr Psychiatry Rep. 2012

6. Schwartz-Lifshitz M, Zalsman G, Giner L, Oquendo MA. Can we really prevent suicide. Curr Psychiatry Rep. 2012

7. Nelson EB. Psychotic depression - beyond the antidepressant/antipsychotic combination. Curr Psychiatry Rep. 2012

8. Murrough JW, Charney DS. Is there anything really novel on the antidepressant horizon. Curr Psychiatry Rep. 2012

9. Pandya M, Altinay M, Malone DA Jr, Anand A. Where in the brain is depression. Curr Psychiatry Rep. 2012 